\title{
Prospective Study of Importance of Clinical Scores in the Differentiation of Stroke Subtypes
}

\author{
Authors \\ Dr Muddada Yugandhar ${ }^{1}$, Dr Balakrishna GT ${ }^{2}$, Dr Gagan Behara ${ }^{3}$, \\ Dr Sarma VSN Rachakonda ${ }^{4}$ \\ ${ }^{1,2}$ Postgraduate, Department of General Medicine, GEMS, Srikakulam \\ ${ }^{3,4}$ Professor, Department of Medicine, GEMS, Srikakulam \\ Corresponding Author \\ Dr Muddada Yugandhar
}

\section{Introduction}

Stroke is defined as "rapidly developing clinical symptoms and/ or signs of focal and at times global (applied to patients in deep coma and to those with subarachnoid hemorrhage) loss of cerebral function with symptoms lasting for more than 24 hours or leading to death, with no apparent cause other than that of vascular origin" . Community based surveys from different region of the country show a crude prevalence rate of strokes presumed to be of vascular origin in the range of 200 per 100000 population ${ }^{2}$. Stroke is the second leading cause of death worldwide, causing 6.2 million deaths in 2011, and is double the rate of heart disease in China. The most important step in the management of acute stroke is differentiating whether the stroke is Ischemic or Hemorrhagic in nature as the management for both is significantly different and management of one subtype is often contraindicated in the other subtype. The most accurate investigation to differentiate between the ischemic or hemorrhagic stroke is Computed Tomography. But the availability of a CT scan in developing countries is limited and whenever available many patients could not be able to afford it. This makes the primary care physicians in acute stroke units resort to clinical features to differentiate between the subtypes. In order to achieve this various clinical score were developed.

In order to help the primary care physicians to effectively differentiate between the stroke subtypes, in 1984, the Guy's Hospital score was developed as a clinical diagnostic tool for intracranial hemorrhage. The calculations involved in Guy's scoring system were too complex for bedside application. Later in 1986, another simpler scoring system was evolved at the Siriraj hospital of Thailand has found greater acceptance. Till such time these scoring system have been fully evaluated and endorsed it is necessary to test them against the gold standard of CT scanning. This study attempts to test the utility of the Guy's Hospital score and Siriraj stroke score in the diagnosis of acute stroke.

\section{Materials and Methods}

The study was done in department of Medicine, Great Eastern Medical School and Hospital. Fifty cases of acute stroke as defined by WHO, 
admitted to medical wards were studied for a duration of one year from December 2017 to December 2018. This is a prospective study.

\section{Method of collection of Data}

Sampling procedure - Purposive sampling

Sample size -50 cases

\section{Inclusion Criteria}

The study group consisted of patients who were admitted to medical wards with a clinical diagnosis of acute stroke as defined by WHO definition.

\section{Exclusion Criteria}

1. Patients with previous history of stroke

2. Patients whose symptoms are suggestive of postictal paralysis

3. Patients with history of trauma to head

4. Subarachnoid hemorrhage

A detailed history and thorough clinical examination were performed at admission and at end of 24 hours. Siriraj stroke score and Guy's Hospital stroke score were assessed. All patients got a CT scan head done within 72 hours of admission into the hospital.

\section{Statistical Analysis}

Both the Siriraj stroke score and Guy's Hospital stroke score were compared with CT findings and sensitivity, specificity and positive predictive value were calculated.

\section{Results and Analysis}

Fifty cases of acute stroke as defined by WHO definition of stroke admitted to the Department of Medicine, Great Eastern Medical School and college, Srikakulam during the period of 20172018 were studied. Siriraj stroke score and Guy's Hospital stroke score were calculated at the admission and after 24hours respectively and correlated with CT scan.

The results of the study are as follows:

Table 1: Siriraj Stroke Score and CT Correlation

\begin{tabular}{|l|c|c|}
\hline \multirow{2}{*}{$\begin{array}{l}\text { Siriraj Stroke } \\
\text { Score }\end{array}$} & \multicolumn{2}{|c|}{ CT Scan diagnosis } \\
\cline { 2 - 3 } & Infarction & Hemorrhage \\
\hline$<-1$ & 8 & 2 \\
\hline-1 to +1 & 11 & 2 \\
\hline$>+1$ & 4 & 23 \\
\hline Total $(\mathrm{n}=50)$ & 23 & 27 \\
\hline
\end{tabular}

\section{Figure 1}

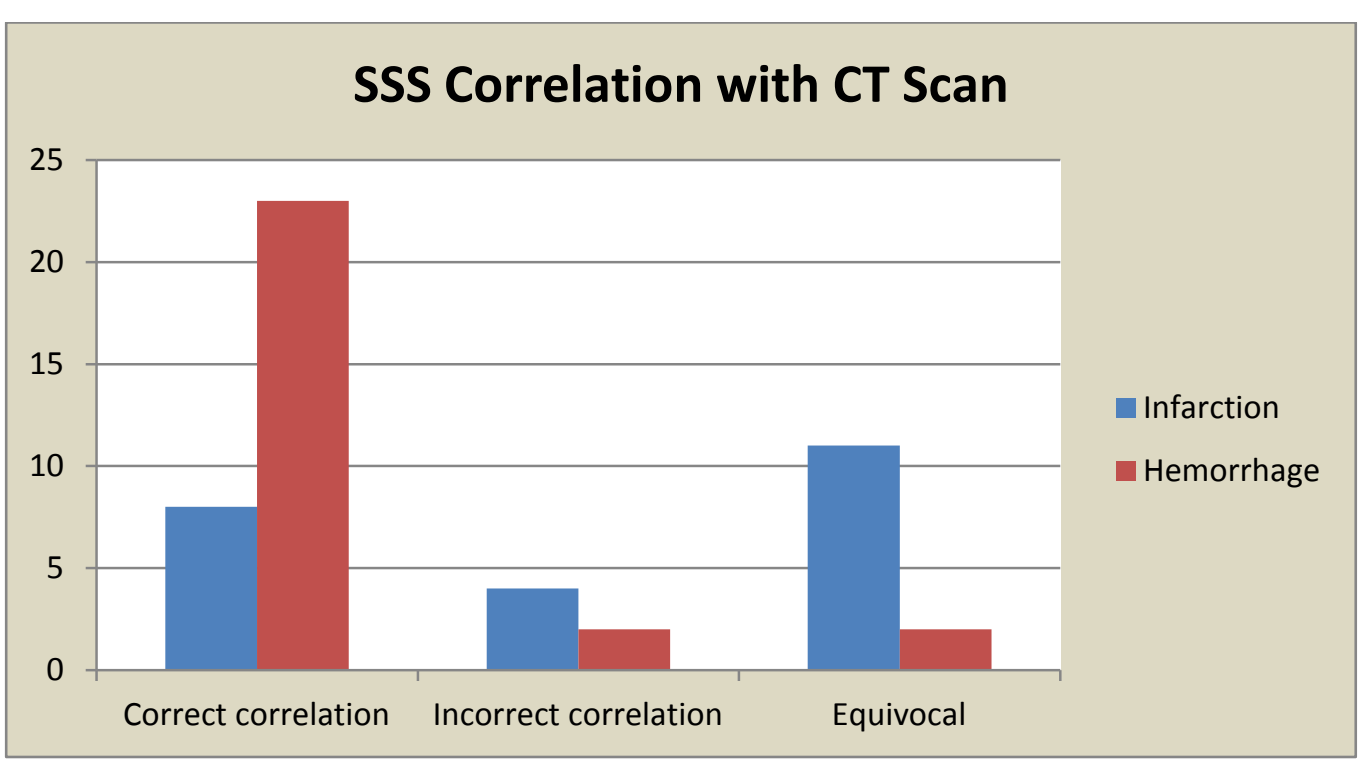

Out of 23 patients diagnosed as infarction by CT scan head, Siriraj stroke score was suggestive of infarction in 8 cases, 11 cases were equivocal and it wrongly diagnosed 4 case of infarction as hemorrhage.

The sensitivity of Siriraj score for ischemic stroke is $66.64 \%$ specificity is $92 \%$, positive predictive value of $80 \%$ and negative predictive value was $85 \%$

Out of 27 patients diagnosed as hemorrhage by CT scan head Siriraj stroke score was suggestive of hemorrhage in 23 cases. In 2 cases it was 
equivocal and it wrongly diagnosed 2 cases of hemorrhage as infarction.

The sensitivity of Siriraj score for hemorrhage is 92\% specificity is $75 \%$ and positive predictive value of $85.19 \%$ and negative predictive value was $85.71 \%$.
Table 2: Guy's Hospital Stroke Score and CT Correlation

\begin{tabular}{|l|c|c|}
\hline \multirow{2}{*}{ Guy's score } & \multicolumn{2}{|c|}{ CT Scan diagnosis } \\
\cline { 2 - 3 } & Infarction & Haemorrhage \\
\hline$<4$ & 9 & 0 \\
\hline 4 to24 & 13 & 7 \\
\hline$>24$ & 1 & 20 \\
\hline Total $(\mathrm{n}=50)$ & 23 & 27 \\
\hline
\end{tabular}

\section{Figure 2}

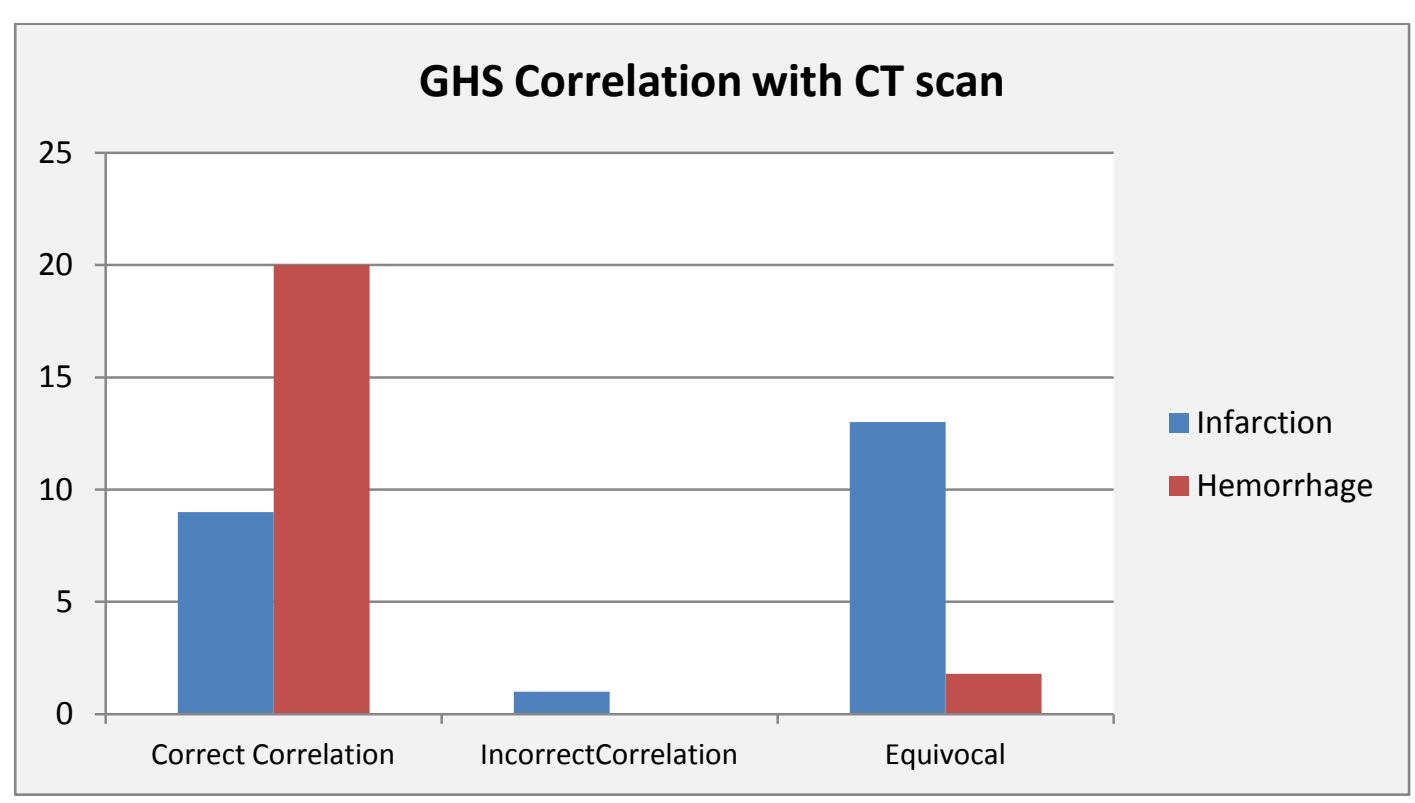

Out of 23 patients diagnosed as infarction by CT scan head Guy's hospital stroke score was suggestive of infarction in 9 cases. In 13cases the score was equivocal and 1 case was wrongly diagnosed as hemorrhage.

The sensitivity of guys hospital stroke score for ischemic stroke is $90 \%$, specificity is $100 \%$ and positive predictive value of $100 \%$ and negative predictive value was $96.43 \%$.
Out of 27 patients diagnosed as hemorrhage by CT scan head Guy's hospital stroke score was suggestive of hemorrhage in 20 cases. In

7 cases the score was equivocal.

The sensitivity of Guy's score for hemorrhagic stroke is $94.46 \%$, specificity is $93.75 \%$ and positive predictive value is $95.24 \%$ and negative predictive value is $100 \%$.

Table 3: Comparison of this study with previous studies

\begin{tabular}{|l|c|c|c|c|}
\hline Author & \multicolumn{2}{|c|}{ INFARCTION } & \multicolumn{2}{c|}{ HEMORRHAGE } \\
\hline & $\begin{array}{c}\text { SENSITIVITY } \\
(\%)\end{array}$ & $\begin{array}{c}\text { SPECIFICITY } \\
(\%)\end{array}$ & $\begin{array}{c}\text { SENSITIVITY } \\
(\%)\end{array}$ & $\begin{array}{c}\text { SPECIFICITY } \\
(\%)\end{array}$ \\
\hline SIRIRAJ STROKE SCORE & & & & 88 \\
\hline Clifford et al $^{5}$ & 69 & 83 & 77.27 & 87.93 \\
\hline Pavan MR et al $^{6}$ & 87.93 & 77.27 & 48 & 85 \\
\hline Hawkins et al $^{7}$ & 61 & 74 & 85 & 73 \\
\hline Kochar et al $^{8}$ & 73 & 85 & 92 & 75 \\
\hline Present study $^{\text {GUYS HOSPITAL SCORING }}$ & 66.64 & 92 & 54 & 89 \\
\hline Clifford et al & & & 80 & 94.54 \\
\hline Pavan MR et al & 70 & 79 & 31 & 95 \\
\hline Hawkins et al & & 80 & 60 & 91 \\
\hline Kochar et al & 94.54 & 70 & 94.46 & 93.75 \\
\hline Present study & 78 & 60 & 100 & \\
\hline
\end{tabular}


In the current study, of both the scores guys hospital stroke score is more sensitive and specific than Siriraj stroke score in the diagnosis of infarction. However, Siriraj stroke score is easy to calculate and the time of assessment is earlier than Guys Hospital stroke score.

In the diagnosis of hemorrhage also Guys hospital stroke score is more sensitive and specific than Siriraj stroke score. Among both the scores Guys hospital stroke appears more reliable than Siriraj stroke score, but the ease of assessment and the time of application is better in case of Siriraj stroke score. In view of above facts, whenever a primary care physician wants to make a decision based on clinical scores, it is more justifiable to use Siriraj stroke score rather than guys hospital stroke score.

But when compared to Computed Tomography both the scores lack enough sensitivity, specificity and positive predictive values. So, whenever a CT scan is available the diagnosis and differentiation of acute stroke and its subtype should be made using a CT scan. In cases where this is not possible, the primary care physician can depend on siriraj stroke score for the diagnosis and differentiation.

Both the siriraj stroke score and guys hospital score are based on clinical features. One important fallacy in both scores is lack formal definitions for certain variables particularly the one "Level of consciousness". It is an important variable and weighing factor in both the scores. An altered level of consciousness can occur both in ischemic and hemorrhagic stroke with more propensity in hemorrhagic stroke, whereas large ischemic strokes can cause cerebral edema and leads to midline shift, brainstem compression, alteration in the level of consciousness and gives false interpretation of hemorrhage. Also the site of infarct can also affect the level of consciousness profoundly when it affects the reticular activating system even though if it is a small infarct. Small infarcts in brainstem and cerebellum can leads to herniation of brainstem with profound loss of consciousness leading to false interpretation as hemorrhage as higher scores will favours a diagnosis of hemorrhagic stroke.

Both SSS and Guys Hospital scoring tend to classify severe strokes as hemorrhagic strokes and strokes of less severity as ischemic regardless of their etiology, therefore both scoring methods needs some modifications in their variables and those variables should be well defined.

Hypertension is one of the most important risk factor for stroke, and at the same time an acute ischemic stroke can cause sudden increase in blood pressure through cerebral autoregulation to increase the cerebral perfusion. This increase in blood pressure favours the diagnosis of hemorrhagic stroke. So therefore both the scores have an over tendency to diagnose a hemorrhagic stroke.

Using both the scores might slightly increase the accuracy of the diagnosis, however it is a time consuming and laborious process. And also, guys hospital score includes almost all the variables included in siriraj stroke score.

However, it is not likely that any score will replace imaging. Where it is impossible to avail a CT scan, and the treatment of stroke therefore limited, health services should lay emphasis on strategies to reduce the risk of stroke in their population.

In the current study Guys hospital score is better than siriraj score in differentiating ischemic stroke from hemorrhagic stroke but the difference between the two scores is no that significant. However both the scores lack enough sensitivity, specificity and positive predictive value when compared to CT scan. Even though Guys hospital score is better than siriraj score, but both of them lack enough sensitivity, specificity and positive predictive value when compares to a CT scan.

So whenever available a CT scan should be done to differentiate the stroke subtype, but when it is not possible, a clinician can use Siriraj score because the two scores doesn't significantly differ and SSS is easy to calculate. 


\section{Limitations}

1. Sample size is too small to generalize the results to a larger population.

2. This study was done in a tertiary care hospital, so the results may not be applicable to patients of Acute Stroke in primary care centres.

3. The prevalence rate of stroke subtypes varies from one set of population to others and hence the results may not be applicable to the general population.

\section{Conclusion}

Computed tomography is the gold standard in the differentiation of infarction from hemorrhage.

Whenever CT scan is not readily available and the physician wished to initiate antithrombotic or antiplatelet treatment, he can use Siriraj score or Guys hospital score with reasonable amount of accuracy.

Of both scores Guys hospital score is more accurate, but Siriraj score is easy to calculate and there is no delay in diagnosis as in case of Guys hospital score. However there is no significant difference between the two scores.

When compared to CT scan, both the scores lack enough accuracy in the differentiation of infarction from hemorrhage.

\section{References}

1. Hatano S. Experience from a multicentre stroke register: a preliminary report. Bulletin of the World Health Organization. 1976;54(5):541.

2. Dalal PM. Neurology, India 1997; 45: 127-131.

3. Mwita C, Kajia D, Gwer S, Etyang A, Newton C. Clinical stroke scores for distinguishing stroke subtypes: a systematic review of diagnostic test accuracy. JBI Database of Systematic Reviews and Implementation Reports. 2013 Mar 1;11(3):191-226.

\section{Pavan}

Manibettu

Raghuram, Mallanagouda Shivanagouda Biradar, Jayakumar Jeganathan. Comparison of the Siriraj stroke Score and the Guy's Hospital Score in South India. Journal of Clinical and Diagnostic Research. 2012 June, Vol6(5): 851-854.

5. Hawkins GC, Bonita R, Broad JB, Anderson NE. Inadequacy of clinical scoring systems to differentiate stroke subtypes in population-based studies. Stroke. 1995 Aug 1;26(8):1338-42.

6. Kochar DK, et al. Poor diagnostic accuracy and applicability of Siriraj stroke score, Allen score and their combination in differentiating acute haemorrhagic and thrombotic stroke. The Journal of the Association of Physicians of India. 2000 Jun;48(6):584-8. 